\title{
Precise Positioning and Orientation of Nanowire Lasers in Regular and Patterned Surfaces
}

\author{
D. Jevtics ${ }^{1 *}$, A. Hurtado ${ }^{1}$, B. Guilhabert ${ }^{1}$, M. Jankauskas ${ }^{1}$, N. Laurand ${ }^{1}$, Q. Gao ${ }^{2}$, H. H. Tan ${ }^{2}$, C. Jagadish ${ }^{2}$ and M. D. Dawson ${ }^{1}$ \\ ${ }^{1}$ Institute of Photonics, SUPA Department of Physics, University of Strathclyde, Technology and Innovation Centre, 99 George \\ Street, G1 1RD, Glasgow, United Kingdom, email dimitars.jevtics@ strath.ac.uk \\ ${ }^{2}$ Department of Electronic Materials Engineering, Research School of Physics and Engineering, The Australian National University, \\ Canberra, ACT 2601, Australia.
}

Abstract - Accurate integration of nanowire (NW) lasers with precise (sub-micrometric) control of their position and orientation angle with other photonic elements is demonstrated by means of a transfer-printing (TP) technique. 1- and 2dimensional arrays of NW lasers are fabricated onto regular and diffractive photonic substrates with full control on the number of NWs in the arrays, their position, orientation angle and spacing between elements.

\section{INTRODUCTION}

Since their first demonstration [1], semiconductor nanowire (NW) lasers have revolutionized photonic technologies offering coherent light sources at the nanoscale. Their unique properties including ultra-small dimensions, highly localized emission, low lasing thresholds, ensure that NW lasers will play a key role in next generation photonic circuitry for a wide range of applications, e.g. biosensors, solid-state electronics, optical communication systems, to name a few [2-4]. However, the difficulty to integrate these nano-lasers from their growth (native) substrate onto selected locations on desired photonic platforms has remained a fundamental problem preventing their widespread introduction into functional optoelectronic products. One of the critical difficulties is the achievement of accurate positioning (with sub-micrometric precision) of NW lasers onto target substrates whilst controlling also their orientation angle. By overcoming this challenge, it would be possible to create a wide range of complex nanophotonic integrated systems with these nano-scale coherent light sources. Several methods of organizing NWs with targeted orientations have been explored and reported in the literature such as: microtip-based systems [5], optical tweezers [6], and contact printing [7]. Although these techniques provide effective solutions to some of the integration difficulties, none of them offers positioning and alignment of individual NWs with high precision onto heterogeneous substrates, whilst retaining prospects for highscale device integration.

To solve this pressing problem limiting the development of functional systems with key-enabling semiconductor NW devices, our research has focused on using a technique usually referred to as transfer-printing (TP) [2][8,9]. This technique utilizes polymer stamps to controllably capture and release semiconductor devices and has been previously used for optoelectronic systems assembly. For instance, transferprinting protocols have been widely used to integrate microLEDs [10,11], III-V semiconductor components onto bespoke photonic platforms [12-14] and recently also to successfully transfer novel materials (e.g. $\mathrm{MoS}_{2}$ [15], graphene [16]). This transfer-printing technique offers therefore great perspectives for the hybrid integration of pre-fabricated devices onto targetsurfaces. This will in turn open great possibilities to assemble functional photonic devices using heterogeneous photonic components as building blocks allowing the development of photonic circuitry that could combine different material groups on a single photonic chip. For example, recent works have used transfer-printing protocols to integrate multiple-color microlight emitting diodes on a simple platform for next-generation visible light communications systems [10]. Besides that, this technique offers nearly $100 \%$ transfer yields [17], thus building great confidence on its future adoption as a main manufacturing technique for next generation integrated optoelectronic systems.

Recently, we have focused on extending the capabilities of this transfer-printing technique to operate with nanoscale devices, such as NW lasers. Using TP we have recently demonstrated [2][9][13,14] controlled positioning of NW lasers onto various substrates (e.g. silica, gold, polymers), as well as the integration of NW lasers onto mechanically flexible platforms which incorporate waveguide circuits that successful coupled and guided the NW laser beams on-chip [13]. Additionally, in a recent work we demonstrated the integration of individual NW lasers onto 'Cat's eye' nano-antennas for enhanced vertical light emission [14].

In this work, we have advanced this fabrication technique at the nanoscale to allow the controllable angular orientation of selected NWs during the transfer-printing processes, whilst maintaining its sub-micrometric positioning accuracy during the NW capture and release stages. Importantly, the TP technique does not degrade the device performance or structure; hence, proving a very useful tool for the hybrid integration of NW lasers onto heterogeneous platforms for functional nanophotonic applications [2].

In this work we have used indium phosphide (InP) NW lasers [2][18] (with dimensions of $435 \mathrm{~nm}$ in diameter and 5-7 $\mu \mathrm{m}$ in length) to demonstrate the potentials of the aforementioned TP technique to build 1-D and 2-D arrays of NW lasers using these ultrasmall nano-lasers as building blocks. These have been built with highly accurate control of NW positioning and orientation angle down to sub-micrometric precision and on a diverse set of substrates with various photonic functions. These include regular 'flat' surfaces, such as silicon or polymers, and patterned diffractive substrates (e.g. polymer gratings). The 
latter provides a first proof-of-concept demonstration towards practical nano-laser systems with tailored emission properties by precisely aligning NW lasers with diffractive nano-photonic structures.

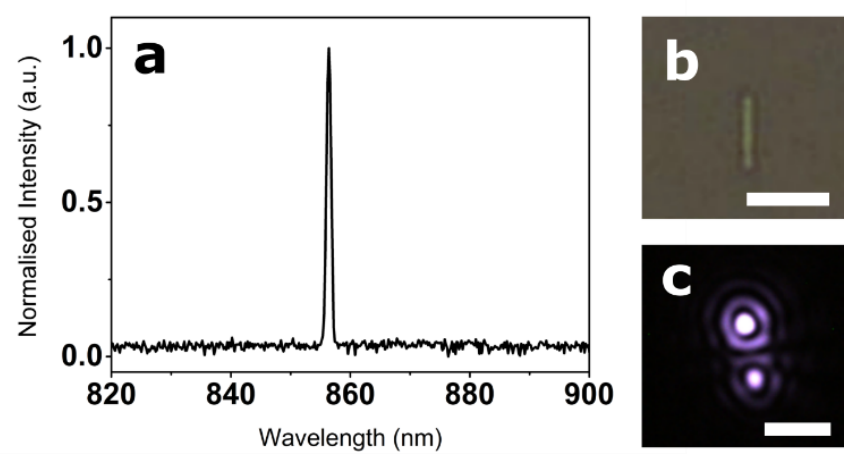

Fig. 1: (a) Spectrum of a typical InP NW laser at room temperature. (b) Bright-field micrograph of a NW laser on a silica substrate. (c) Dark-field micrograph of the NW laser under optical excitation above threshold. Scale bars: $5 \mu \mathrm{m}$.

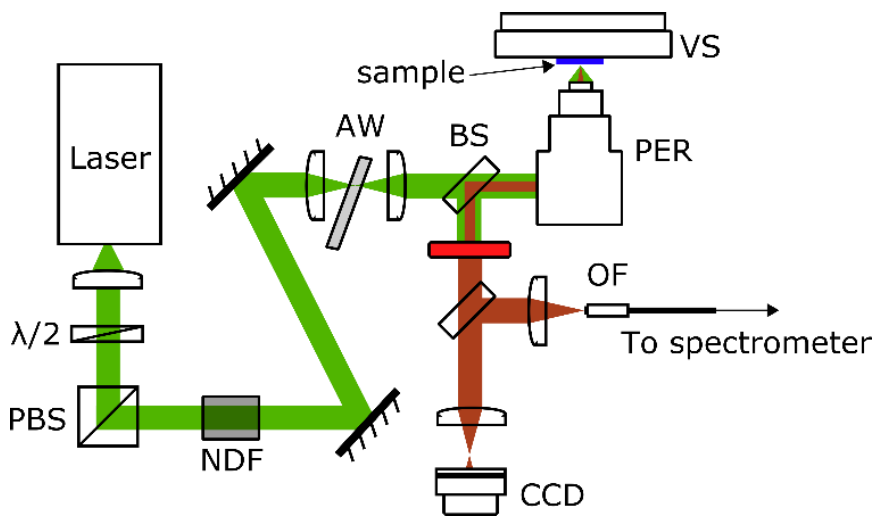

Fig. 2 : Micro-PL setup. $\lambda / 2=$ half-wave plate, $\mathrm{PBS}=$ polarizing beam splitter, $\mathrm{NDF}=$ neutral density filter, $\mathrm{AW}=$ attenuation wheel, $\mathrm{BS}=$ beam splitter, $\mathrm{PER}=$ periscope with optical objective, $\mathrm{VS}=$ variable stage, $\mathrm{OF}=$ optical fiber, $\mathrm{CCD}=\mathrm{CCD}$ camera

\section{CHARACTERISATION SETUP}

The InP NW lasers used in this work have typical lasing emissions in the spectral range from 840 to $900 \mathrm{~nm}$. Fig. 1(a) shows the measured room-temperature optical spectrum from a typical InP NW laser lying down on a silica substrate under optical excitation above its lasing threshold. It is worth mentioning that although the spectrum for this specific InP NW laser shows single mode emission, these devices can support multiple transverse modes (for a review on the modal and light emission properties of these nano-lasers see [19]). Fig. 1(b) and (c), respectively show the bright- and dark-field micrographs of the NW laser whose spectrum is shown in Fig. 1(a). To characterize the room-temperature emission of the InP NW lasers of this work we used a micro-photoluminescence (PL) setup to optically pump the NW lasers [2]. Fig. 2 shows the schematic of the micro-PL setup. This allows to collect light using a CCD camera and measure the optical spectra simultaneously; thereby providing the lasing threshold and spectral properties of NW lasers as well as their emission features. A green pulsed laser (pulse width $0.75 \mathrm{~ns}$ and repetition rate of $7.2 \mathrm{kHz}$ ) at the wavelength of $532 \mathrm{~nm}$ is used as the optical pump and the beam is directed onto the NWs through a combination of free-space, a periscope system and a 10x optical objective. Light emission from the NW lasers is collected by a high-resolution spectrometer with a spectral resolution down to $50 \mathrm{pm}$.
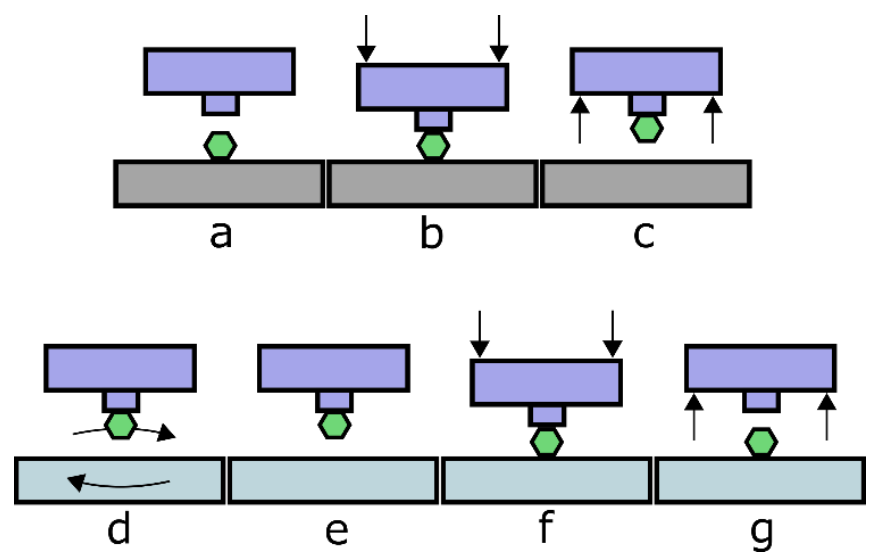

Fig. 3: (a) - (g) Stages of the transfer-printing technique for the positioning and orientation of NW lasers. (a) The micro-stamp is aligned with a selected NW. (b) The tip of the micro-stamp makes full surface contact with the NW. (c) The NW is captured. (d) The microstamp (with the NW) is moved onto the target area on the receiving substrate. The stage is rotated to the specified orientation of the NW. (e) The micro-stamp is accurately aligned to the target position in the receiver surface. (f) Full surface contact between micro-stamp and receiver substrate. $(\mathrm{g})$ The $\mathrm{NW}$ is released on the target location with the desired orientation in the receiver substrate.

\section{TRANSFER-PRINTING TECHNIQUE}

The transfer-printing (TP) technique, firstly reported by Prof J. Rogers' Group at the University of Illinois (for a review see [8]), relies on principles of reversible adhesion [20]. It allows the controllable pick up of micro-/nano-scale devices from a "donor" substrate and placing them at desired locations onto a target receiver surface. These pick-and-place processes are carried out by means of elastomeric $\mu$-stamps [2], made of polydimethylsiloxane (PDMS), a widely used transparent silicone rubber. In this work, the TP technique is extended to allow the printing of ultra-small NW lasers with controlled position and orientation by means of a modified dip-pen nanolithography system [2], which has a three-axis translation stage, pitch and yaw control and a rotational module to offer 6 degrees of freedom for NW capture and release. This allows not only to transfer a NW with high degree of accuracy from its original 'donor' substrate onto a secondary 'receiver' surface, but also to fully rotate the nano-lasers during the release stage for increased accuracy and versatility.

The stages of the TP technique are depicted in Figs. 3 (ag). At first, the micro-stamp is aligned with a selected NW laser (Fig. 3(a)). Then, after making surface contact, the NW laser is captured by the micro-stamp (Figs. 3(b-c)). Next, the 
micro-stamp is controllably moved onto a selected position on the receiver surface (which could be any surface of choice, such as polymer, silica, silicon, metals, etc.). The stage can be then rotated to match a selected release orientation angle between NW and receiving substrate (Fig. 3(d)), after which the micro-stamp is aligned at the target location (Fig. 3(e)). Full surface contact is made between NW and receiving substrate (see Fig. 3(f)). Finally, the NW is released onto the target location with high-accuracy (sub-micrometric precision), as shown in Fig. 3(g). Full details on the individual stages of the transfer-printing technique of this work for the precise positioning and orientation of $\mathrm{NW}$ lasers can be found in [2][13].
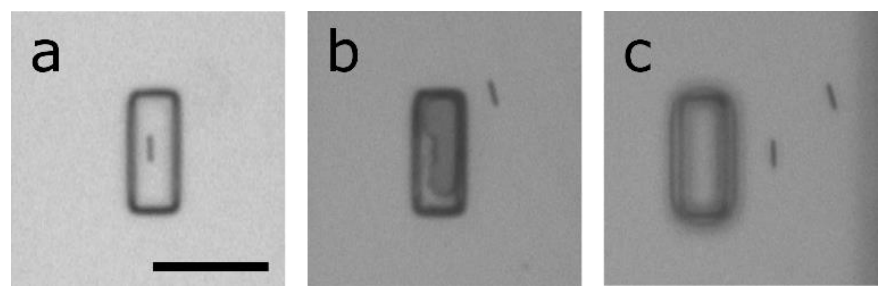

Fig. 4: (a) - (c) Images showing the transfer-printing stages of an InP NW laser: (a) The micro-stamp captures an InP NW laser from the donor substrate. (b) Surface contact is made between micro-stamp and receiving substrate to release the NW. (c) NW is positioned at the desired location on the receiving substrate. Scale bar: $30 \mu \mathrm{m}$.

\section{RESULTS}

The NWs used in this work themselves form a Fabry-Perot cavity that can therefore achieve lasing emission from their endfacets when optically excited [18]. The InP NW lasers were mechanically transferred from their growth sample [2] onto a donor substrate of choice (in this case PDMS).

Figs. 4(a-c) depict in detail the process followed to transferprint one of the NW lasers included in the 1D array shown in Fig. 5 (marked by a red dotted square). The array was built by means of TP on a silicon surface using a micro-stamp design with flat tip [2]. It is worth noting that using flat-tip microstamps is important for the fabrication of systems where the orientation angle of the NWs is crucial. Since the micro-stamp design is fully transparent (in contrast to other semi-transparent types [2-9]), it allows us to clearly visualize all the transferprinting processes to achieve a higher degree of precision. Fig. 4(c) shows that after the transfer-printing protocols, the selected NW laser is positioned at a desired location with a controlled orientation angle of 15 degrees with respect to another previously transfer-printed NW laser $20 \mu \mathrm{m}$ apart. Repeating this process, we successfully built a 1-Dimensional NW laser array of Fig. 5 The NW lasers in the array have a spacing of 20 $\mu \mathrm{m}$ and sequentially rotated by 15 degrees with respect to each other. Importantly, this transfer-printing technique allows precision levels well below $1 \mu \mathrm{m}$, as initially investigated in [13], where axis alignment accuracies of $50 \pm 35 \mathrm{~nm}$ were achieved for two NW lasers printed in series.

In this work, we have also developed 2-Dimensional (2-D) arrays with NW lasers where both the position and the orientation angle of the individual NW devices are fully controlled. Fig. 6(a) shows an SEM image of a 2-D 3 x 3 array of NW lasers built on a silicon substrate with lateral (vertical) separation between contiguous elements in the same row (column) equal to $10 \mu \mathrm{m}$. In the array of Fig. 6(a), the NWs in each column are deposited with distinct angular orientations, namely 0 (left), 45 (middle) and 90 (right) degrees.

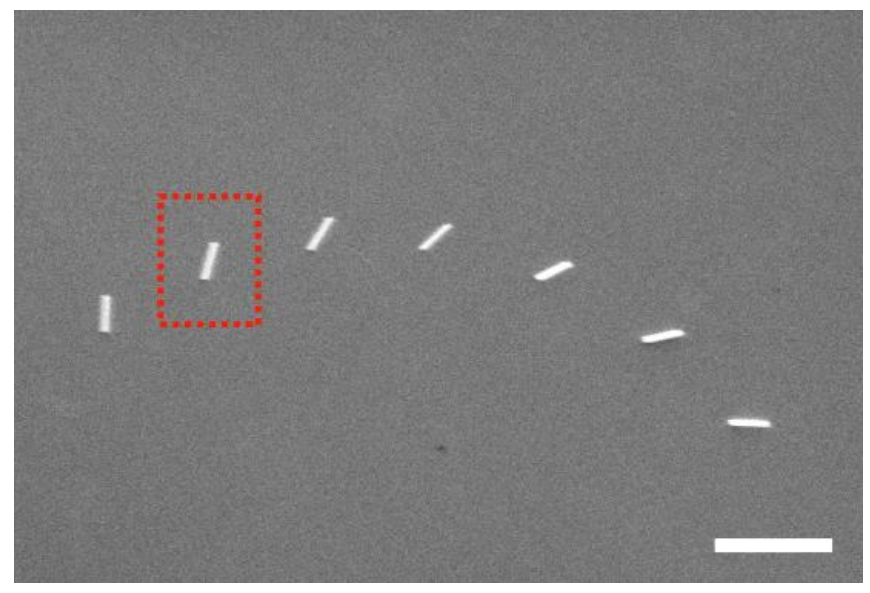

Fig. 5: Scanning Electron Microscope (SEM) image of a 1-D array of NW lasers with individual elements separated by $20 \mu \mathrm{m}$ and with controlled orientation angles from 0 to 90 degrees (in 15 degrees steps). Scale bar: $20 \mu \mathrm{m}$.

Fig. 6(b) shows our first results on this new research direction demonstrating the accurate integration of InP NW lasers at specific locations and desired orientation angle $(0,45$, and 90 degrees) onto a $50 \mathrm{~nm}$-pitch polymer grating (fabricated in PDMS). Fig. 6(b) provides our first proof-of-concept demonstration on the controllable integration of semiconductor NW lasers onto grating structures with their axes fully or partially aligned (and misaligned) with respect to the grating's orientation (marked by the double black arrow in Fig. 6(b)). This will in turn affect differently the distinct modes supported by the NW lasers. The proof-of-concept demonstrations discussed here show indeed excellent prospects for future systems with NW lasers controllably integrated onto patterned diffractive substrates (e.g. nanoantennas, polymer gratings, etc.) to enable us to tailor the emission properties of NW lasers.
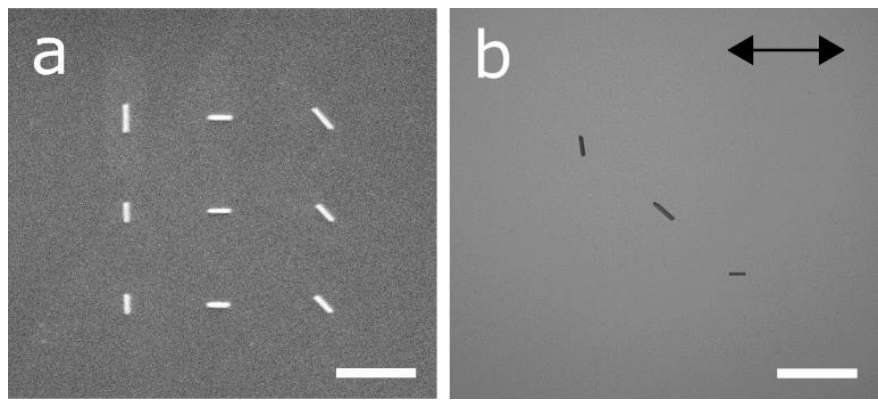

Fig. 6: (a) SEM image of a 2D array of InP NW lasers. The NWs on different columns have been integrated with different angle orientations (0, 45 and 90 degrees). Scale bar: $20 \mu \mathrm{m}$. (b) NW lasers transfer-printed on a $50 \mathrm{~nm}$-pitch PDMS grating with different orientation angles (0, 45 and 90 degrees). Scale bar: $30 \mu \mathrm{m}$. 


\section{CONCLUSIONS}

This work demonstrates the accurate positioning of semiconductor NW lasers with full control of their orientation angle onto diverse substrates by means of a nanoscale transferprinting technique. We fabricated 1-D and 2-D arrays of NW lasers with controlled separation between elements and desired NW orientation angles. Our technique also offers exciting prospects for the fabrication of nano-laser systems where both the position and orientation of the NWs are critical for optimized performance. In particular, we provide proof-ofconcept demonstration of precise integration of NW lasers at desired locations and with controlled orientation angle onto polymer grating substrates for future nano-laser systems with tailored emission wavelength.

\section{ACKNOWLEDGMENT}

The authors would like to acknowledge funding by the Australian Research Council and the UK EPSRC Doctoral Training Partnership. The Australian National Fabrication Facility is acknowledged for access to their growth facilities.

\section{REFERENCES}

[1] M. H. Huang, S. Mao, H. Feick, H. Yan, Y. Wu, H. Kind, E. Weber, R. Russo, P. Yang, "Room-Temperature Ultraviolet Nanowire Nanolasers," Science, vol. 292, no. 5523, pp. 1897-1899, Jun. 2001

[2] B. Guilhabert, A. Hurtado, D. Jevtics, Q. Gao, H. H. Tan, C. Jagadish, and M. D. Dawson, "Transfer Printing of Semiconductor Nanowires with Lasing Emission for Controllable Nanophotonic Device Fabrication," ACS Nano, vol. 10, no. 4, pp. 3951-3958, Mar. 2016

[3] S. W. Eaton, A. Fu, A. B. Wong, C.-Z. Ning, and P. Yang, "Semiconductor nanowire lasers," Nature Reviews Materials, vol. 1, no. 6, pp 1-11, May 2016

[4] R. Röder and C. Ronning, "Review on the dynamics of semiconductor nanowire lasers," Semiconductor Science and Technology, vol. 33, no. 3, 033001, Feb. 2018

[5] J. B. Wright, S. Campione, S. Liu, J. A. Martinez, H. Xu, T. S. Luk, Q. Li, G. T. Wang, B. S. Swartzentruber, L. F. Lester, and I. Brener, "Distributed feedback gallium nitride nanowire lasers," Applied Physics Letters, vol. 104, no. 4, 041107, Jan. 2014

[6] Z. Yan, M. Pelton, L. Vigderman, E. R. Zubarev, and N. F. Scherer, "Why Single-Beam Optical Tweezers Trap Gold Nanowires in Three Dimensions," ACS Nano, vol. 7, no. 10, pp. 8794-8800, Sep. 2013.

[7] D. Roßkopf and S. Strehle, "Surface-controlled contact printing for nanowire device fabrication on a large scale," Nanotechnology, vol. 27, no. 18, 185301, Mar. 2016

[8] A. Carlson, A. M. Bowen, Y. Huang, R. G. Nuzzo, and J. A. Rogers, "Transfer Printing Techniques for Materials Assembly and Micro/Nanodevice Fabrication," Advanced Materials, vol. 24, no. 39, pp. 5284-5318, Aug. 2012

[9] A. Hurtado, D. Jevtics, B. Guilhabert, Q. Gao, H. H. Tan, C. Jagadish, M. D. Dawson, "Transfer printing of semiconductor nanowire lasers," IET Optoelectronics, vol. 12, no. 1, pp. 30-35, Feb. 2018

[10] K. Rae, E. Xie, C. Foucher, B. Guilhabert, R. Ferriera, D. Zhu, D.J. Wallis, C.J. Humphreys, R.A. Oliver, E. Gu, N. Laurand, and M.D. Dawson, "Transfer Printed Multi-color Integrated Devices for Visible Light Communication Applications," in Light, Energy and the Environment, 2016

[11] A. J. Trindade, B. Guilhabert, D. Massoubre, D. Zhu, N. Laurand, E. Gu, I. M. Watson, C. J. Humphreys, and M. D. Dawson, "Nanoscaleaccuracy transfer printing of ultra-thin AlInGaN light-emitting diodes onto mechanically flexible substrates," Applied Physics Letters, vol. 103 , no. 25 , p. 253302 , Dec. 2013

[12] C. Klitis, B. Guilhabert, J. McPhillimy, S. May, N. Zhang, M. J. Strain, and M. Sorel, "Transfer printing of photonic nanostructures to silicon integrated circuits," in Conference on Lasers and Electro-Optics, 2018.

[13] D. Jevtics, A. Hurtado, B. Guilhabert, J. McPhillimy, G. Cantarella, Q.
Gao, H. H. Tan, C. Jagadish, M. J. Strain, and M. D. Dawson, "Integration of Semiconductor Nanowire Lasers with Polymeric Waveguide Devices on a Mechanically Flexible Substrate," Nano Letters, vol. 17 , no. 10, pp. 5990-5994, Sep. 2017

[14] W.-Z. Xu, F.-F. Ren, D. Jevtics, A. Hurtado, L. Li, Q. Gao, J. Ye, F. Wang, B. Guilhabert, L. Fu, H. Lu, R. Zhang, H. H. Tan, M. D. Dawson, and C. Jagadish, "Vertically Emitting Indium Phosphide Nanowire Lasers," Nano Letters, vol. 18, no. 6, pp. 3414-3420, May 2018.

[15] H. Jia, R. Yang, A. E. Nguyen, S. N. Alvillar, S. Bobek, M. Wurch, C.-Y. Huang, L. Bartels, and P. X.-L. Feng, "Single- and few-layer transferprinted CVD MoS2 nanomechanical resonators with enhancement by thermal annealing," in 2016 IEEE International Frequency Control Symposium (IFCS), 2016.

[16] D. Song, A. Mahajan, E. B. Secor, M. C. Hersam, L. F. Francis, and C. D. Frisbie, "High-Resolution Transfer Printing of Graphene Lines for Fully Printed, Flexible Electronics," ACS Nano, vol. 11, no. 7, pp. 74317439, Jul. 2017.

[17] D. Gomez, K. Ghosal, T. Moore, M. A. Meitl, S. Bonafede, C. Prevatte, E. Radauscher, A. J. Trindade, and C. A. Bower, "Scalability and Yield in Elastomer Stamp Micro-Transfer-Printing," in 2017 IEEE 67th Electronic Components and Technology Conference (ECTC), 2017

[18] Q. Gao, D. Saxena, F. Wang, L. Fu, S. Mokkapati, Y. Guo, L. Li, J. WongLeung, P. Caroff, H. H. Tan, and C. Jagadish, "Selective-Area Epitaxy of Pure Wurtzite InP Nanowires: High Quantum Efficiency and RoomTemperature Lasing," Nano Letters, vol. 14, no. 9, pp. 5206-5211, Aug. 2014

[19] D. Saxena, F. Wang, Q. Gao, S. Mokkapati, H. H. Tan, and C. Jagadish, "Mode Profiling of Semiconductor Nanowire Lasers," Nano Letters, vol. 15, no. 8, pp. 5342-5348, Jul. 2015.

[20] J. Wu, S. Kim, W. Chen, A. Carlson, K.-C. Hwang, Y. Huang and J. A. Rogers, "Mechanics of reversible adhesion," Soft Matter, vol. 7, no. 18, p. $8657-8662$, Sep. 2011 